
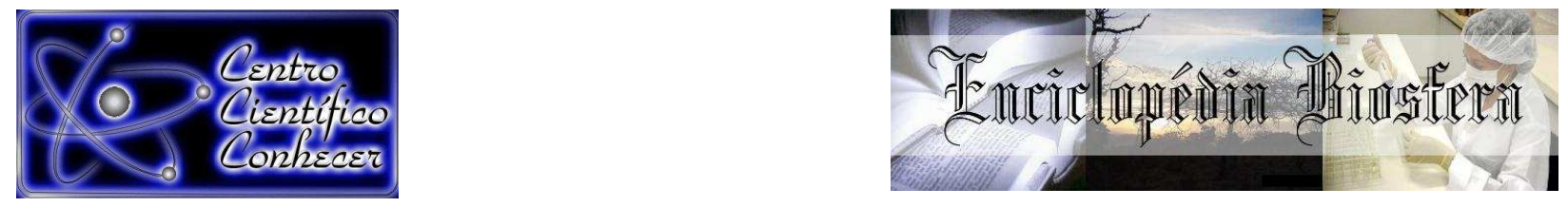

\title{
DENSIDADE BÁSICA DA MADEIRA DE SCHINUS TEREBINTHIFOLIUS RADDI EM POVOAMENTO PURO NO MUNICÍPIO DE PINHAIS - PR
}

\author{
Mateus Niroh Inoue Sanquetta ${ }^{1}$; Carlos Roberto Sanquetta ${ }^{2}$; Alexandre Baumel dos \\ Santos ${ }^{1}$; Vinicius Morais Coutinho1; Ana Paula Dalla Corte ${ }^{2}$ \\ 1. Graduando em Engenharia Florestal da Universidade Federal do Paraná, \\ Curitiba, Brasil (mateus.sanquetta@hotmail.com) \\ 2. Eng. Florestal, Dr., Professor da Universidade Federal do Paraná \\ Centro BIOFIX de Pesquisa em Biomassa e Sequestro de Carbono \\ Universidade Federal do Paraná - UFPR
}
Recebido em: 08/09/2015 - Aprovado em: 14/11/2015 - Publicado em: 01/12/2015
DOI: http://dx.doi.org/10.18677/Enciclopedia_Biosfera_2015_122

\begin{abstract}
RESUMO
A aroeira-pimenteira possui potencial econômico de uso condimentar dos seus frutos, e também de sua madeira. A densidade básica é uma característica que contribui para a determinação da qualidade da madeira, sendo importante para definição do seu uso. Nesse sentido, a densidade básica da madeira de diferentes alturas do fuste de 30 indivíduos de Schinus terebinthifolius foi determinada em laboratório. Os indivíduos arbóreos em questão advêm de um plantio puro localizado no município de Pinhais-PR, estabelecido em 2003. As análises foram feitas para diferentes porções do fuste, dividindo-o em base, no dap (diâmetro à altura do peito ou a $1,30 \mathrm{~m}$ do solo) e ápice. Os valores médios observados variaram de $0,443 \pm 0,033$ e $0,488 \pm 0,041 \mathrm{~g} \cdot \mathrm{cm}^{-3}$ para o ápice e a base, respectivamente, e de $0,452 \pm 0,026 \mathrm{~g} \cdot \mathrm{cm}^{-3}$ para 0 dap. A densidade básica média para todas as três posições no fuste foi de $0,463 \pm 0,029 \mathrm{~g} \cdot \mathrm{cm}^{-3}$. Conclui-se que há diferença estatística da densidade ao longo do fuste, sendo mais alta na base do que no dap e no topo. Os valores encontrados estão dentro da faixa reportada pela literatura.
\end{abstract}

PALAVRAS-CHAVE: aroeira-vermelha, análise estatística, massa específica, plantação florestal, qualidade da madeira.

\section{WOOD BASIC DENSITY OF Schinus terebinthifolius Raddi IN A PURE STAND AT PINHAIS MUNICIPALITY - PR}

\begin{abstract}
The Brazilian pepper tree has economic potential use of its spicy fruit, and of its wood as well. The basic density is a characteristic that contributes to the determination of wood quality, and therefore to define their use. In this sense, basic wood density of different heights along the stems of 30 individuals of Schinus terebinthifolius was determined in the laboratory. Individual trees under analysis come from a pure plantation located in the municipality of Pinhais-PR, established in 2003. The analyses were performed for different portions of the tree stem, dividing it into base, $d b h$ (diameter at breast height or $1.30 \mathrm{~m}$ above the ground level) and top. The average figures of wood density ranged from $0.443 \pm 0.033$ and $0.488 \pm 0.041$ $\mathrm{g} . \mathrm{cm}^{-3}$ to the top and the base, respectively, and $0.452 \pm 0.026 \mathrm{~g} . \mathrm{cm}^{-3}$ for the $d b h$.
\end{abstract}


The average specific gravity for all three positions in the stem was $0.463 \pm 0.029$ $\mathrm{g} . \mathrm{cm}^{-3}$. It was concluded that there is a statistical difference in basic wood density along the stem, being the value at the base higher than that at $d b h$ and at the top. The values found are within the range reported in the literature.

KEYWORDS: Brazilian pepper tree, statistical analysis, density, plantation forestry, wood quality.

\section{INTRODUÇÃO}

A densidade é uma característica importante da madeira, sendo resultante de vários fatores genéticos e ambientais. Essa grandeza é relevante na caracterização da qualidade da madeira e na determinação de seus usos potenciais. Muitas vezes é expressa como "densidade básica", ou o peso seco dividido pelo volume da madeira saturada de água. A relação entre massa e volume é um dos índices mais simples e importantes para avaliação das propriedades funcionais da madeira, refletindo também diferentes atributos da história de vida de espécies arbóreas (WILLIAMSON \& WIEMANN, 2011).

Além da relevância para o conhecimento da qualidade da madeira, a densidade é uma variável importante para estimativas de estoques de carbono arbóreo em ecossistemas florestais. Seu conhecimento permite particularizar as variações inter e intraespecíficas e ambientais nos estoques e a dinâmica de carbono, tendo aplicação direta nos acordos internacionais sobre mudanças climáticas globais (JATI et al., 2014).

A aroeira-pimenteira (Schinus terebinthifolius Raddi) possui potencial econômico para as regiões onde é encontrada. $\mathrm{O}$ uso dos seus frutos é difundido em nível nacional e internacional como condimentar. No entanto, vem sendo explorada em pequena escala no Brasil, sendo que a colheita da maioria dos frutos é realizada em áreas nativas. Além disso, é utilizada na medicina popular como antitérmica, analgésica, depurativa e no tratamento de doenças do sistema urogenital. Existem relatos de que apresenta atividade antimicrobiana, anti-inflamatória e antiulcerogênica (CARVALHO et al., 2013).

Não obstante os difundidos usos dos seus frutos, a madeira da aroeira também pode ser utilizada para diversas finalidades, como a produção de energia, peças roliças e serradas, entre outras (CARVALHO, 2003). A madeira é resistente, podendo ser utilizada como esteios e mourões, devido à sua durabilidade prolongada (REITZ et al., 1978, SANCHOTENE, 1985). Apesar disso, possui baixo valor comercial, o que pode estar relacionado ao precário conhecimento das características que determinam a sua qualidade.

Desta forma, o presente estudo teve como objetivo a determinação da densidade básica da madeira de árvores plantadas de aroeira, a fim de clarificar e contribuir para o conhecimento da espécie e o emprego de sua madeira, bem como gerar informações que sejam úteis na quantificação de estoques e dinâmica de carbono na espécie.

\section{MATERIAL E MÉTODOS}

\section{Área de estudo}

O presente estudo foi desenvolvido com indivíduos arbóreos da espécie Schinus terebinthifolius provenientes de um plantio experimental localizado na Estação Experimental do Canguiri, no município de Pinhais, região metropolitana de 
Curitiba. O município de Pinhais está situado no Primeiro Planalto Paranaense. Segundo a classificação climática de Köppen, o clima da região é caracterizado como temperado úmido mesotérmico (Cbf). Possui precipitação bem distribuída durante todo 0 ano, não apresenta estação seca definida. Conta também com invernos rigorosos e verão com temperaturas amenas. Coordenadas de latitude $25^{\circ}$ 26' 41"S e longitude 49 11' 33" W, a altitude média encontra-se em torno de 890 metros.

\section{Material}

O plantio em questão é homogêneo, composto por árvores, instalado com o objetivo de desenvolver pesquisas relacionadas à espécie. O plantio destas árvores se deu em 2003 e o espaçamento utilizado foi de $2 \mathrm{~m} \times 2 \mathrm{~m}$.

A aroeira tem como sinonímias aroeira-vermelha, aroeira-pimenteira, entre outros nomes, e pertence à família Anacardiaceae. Ocorre de forma natural e abrangente em todo o território brasileiro, caracterizando-se como uma espécie nativa pioneira presente em vários biomas brasileiros (RIZZINI, 1970). De característica resistente a solos pobres, possui rápido crescimento, além de suportar condições de inundação e apresentar altas taxas de sobrevivência (CORADIN et al., 2011).

Para realização deste estudo foram derrubados 30 indivíduos de $S$. terebinthifolius a fim de se realizar análises de densidade básica da madeira. Desta forma, os fustes derrubados foram segmentados, de onde foram coletados discos representativos de três partes dos mesmos: base, dap (diâmetro à altura do peito ou $1,30 \mathrm{~m}$ do solo) e ápice. A casca presente em cada disco foi retirada e unificada para um estudo posterior. Por fim, o material foi devidamente identificado individualmente e encaminhado para análises de laboratório.

Os dap das árvores amostra deste estudo variaram de 5,00 a $11,60 \mathrm{~cm}$, com média de $8,02 \mathrm{~cm}$. As alturas totais oscilaram entre 6,00 a $8,80 \mathrm{~m}$, com média de $7,52 \mathrm{~m}$.

\section{Análises laboratoriais}

Posteriormente à atividade realizada em campo, o material foi submetido à determinação da densidade básica da madeira, empregando-se o método da balança hidrostática, recomendado NBR 11941 (ABNT, 2003) A metodologia consiste na divisão da massa seca pelo volume saturado, obtido com 0 deslocamento em água. Assim, os discos foram fragmentados em cunhas de forma que as mesmas tivessem forma e peso próximos, a fim de facilitar o emprego da metodologia.

As cunhas foram submetidas a total saturação, submersas em água em um dessecador lacrado a vácuo. Em seguida, pesou se individualmente as cunhas em uma balança hidrostática, a partir do deslocamento em água obtém-se o volume saturado. Finalmente, as cunhas foram depositadas em estufa a $70^{\circ} \mathrm{C}$ até atingir peso constante. Foram realizadas pesagens periodicamente até que se obteve 0 peso seco. A densidade básica foi determinada pela seguinte equação:

$D_{b}=\frac{M_{s}}{V_{u}}$

Em que: 
$D_{b}=$ densidade básica $\left(\mathrm{g} \cdot \mathrm{cm}^{-3}\right)$;

$M_{s}=$ massa seca $(\mathrm{g})$;

$V_{u}=$ volume saturado $\left(\mathrm{cm}^{-3}\right)$.

\section{Análises estatísticas}

Foram realizadas análises estatísticas descritivas dos dados de densidade básica obtidos em laboratório. Subsequentemente, empregaram-se os testes de normalidade de Kolmogorov-Smirnov (D), Cramér-von Mises (W2), Anderson-Darling (A2), Kuiper (V), Watson (U2), Lilliefors (D) e Shapiro-Wilk (W) e de homogeneidade de variância disponíveis no software ASSISTAT. Em sequência, procedeu-se ao teste de Tukey para verificar a existência de diferenças estatísticas significativas entre as densidades nas diferentes posições do fuste.

\section{Outras análises}

Foi realizada a análise da relação entre o dap e a densidade básica. Para esse fim foi calculado o coeficiente de correlação simples de Pearson e elaborado um gráfico de dispersão entre ambas as variáveis.

Análise da distribuição de frequência da variável densidade básica da madeira foi também efetuada, considerando as três posições no fuste: base, dap e ápice.

\section{RESULTADOS E DISCUSSÕES}

Os dados empregados neste estudo mostraram-se distribuídos normalmente, como indicado pelos testes retro citados. Verificou-se também homogeneidade de variância ao nível de $5 \%$ de probabilidade, o que possibilitou a realização da ANOVA e do teste de comparação de médias de Tukey.

A priori, a densidade básica determinada para as três porções do fuste variou em termos médios de $0,443 \mathrm{~g} \cdot \mathrm{cm}^{-3}$ a $0,488 \mathrm{~g} \cdot \mathrm{cm}^{-3}$ para 0 ápice e a base, respectivamente, sendo que para a posição do dap o valor médio encontrado foi de $0,452 \mathrm{~g} \cdot \mathrm{cm}^{-3}$. Com relação às medidas de dispersão (desvio padrão), os valores encontrados foram $\pm 0,033 ; \pm 0,041$ e $\pm 0,026$, respectivamente. As dispersões positivas e negativas em relação às médias são demonstradas graficamente (FIGURA. 1). Os coeficientes de variação foram determinados, obtendo-se $8,43 \%$ e $7,43 \%$ para fuste da base e apical, respectivamente. Já o CV\% para o fuste na porção do dap foi de $5,66 \%$ (Tabela 1).

TABELA 1 - Densidade básica da madeira de amostras tomadas em diferentes porções do fuste de Schinus terebinthifolius em Pinhais-PR

\begin{tabular}{cccccc}
\hline \multirow{2}{*}{ Porção do fuste } & \multicolumn{5}{c}{ Densidade Básica $\left(\mathrm{g} . \mathrm{cm}^{-3}\right)$} \\
\cline { 2 - 6 } & \multicolumn{2}{c}{ Média } & Desvio Padrão & CV\% & $\mathrm{n}$ \\
\hline Base & 0,488 & a & 0,041 & 8,43 & 30 \\
dap & 0,452 & b & 0,026 & 5,66 & 30 \\
Ápice & 0,443 & b & 0,033 & 7,43 & 30 \\
Média & 0,463 & & 0,029 & 6,30 & 30 \\
\hline
\end{tabular}


Apesar da carência de informações científicas a respeito desta característica da madeira de $S$. terebinthifolius, trabalhos são encontrados na literatura. No entanto, por não se tratarem de estudos específicos sobre a densidade básica, estes não destacam a importância da variação da mesma ao longo do fuste.

A determinação da densidade básica deve ser realizada em diferentes alturas do fuste e os valores devem, se possível, serem apresentados separadamente, a fim de clarificar possíveis variações, conforme evidenciado neste estudo. No presente trabalho verificou-se que a densidade básica da madeira tende a decrescer conforme o aumento da altura analisada (Figura 1).

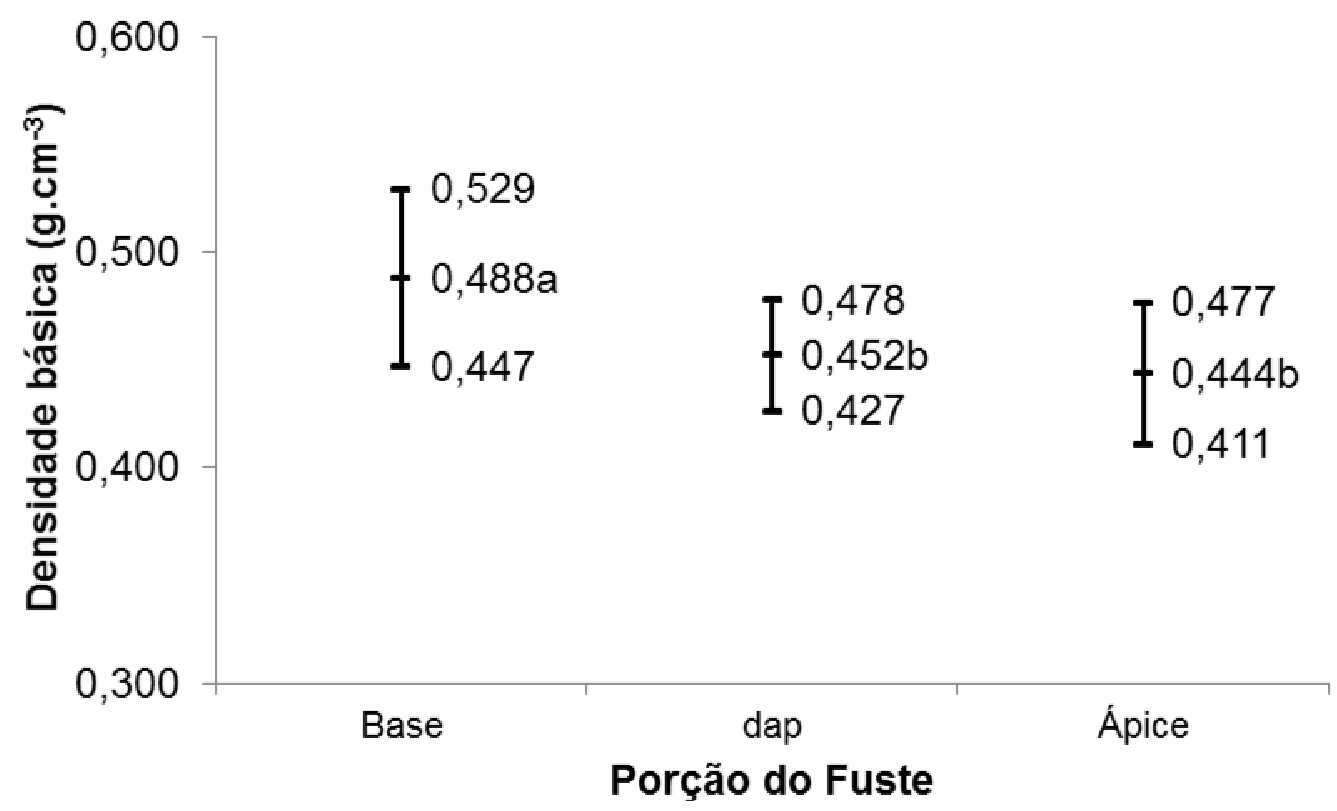

FIGURA 1 - Densidade básica em amostras de madeira de 30 indivíduos de Schinus terebinthifolius plantados em Pinhais-PR

Desta forma, a discussão com outros dados será realizada com uma média geral determinada para a espécie. Autores como BAGGIO (1988), realizaram estudos com amostras de diferentes alturas, mas reportaram apenas valores médios. O autor observou que aos sete anos de idade, árvores de aroeira apresentaram uma densidade básica de $0,493 \mathrm{~g} . \mathrm{cm}^{-3} \mathrm{em}$ amostras compostas em porções tomadas em diferentes alturas, sendo estas de: 1,3; 1,5 e 2,0 metros. Notase que, mesmo tratando-se de indivíduos mais jovens que os aqui analisados, os valores encontrados foram superiores aos encontrados neste estudo. Esta diferença pode ser explicada pelas alturas tomadas nas determinações do autor, também por variações ocasionais que ocorrem de acordo com o espaçamento do plantio, região, idade, entre outros.

Em outro estudo, NOGUEIRA (2010) realizou análises de densidade considerando diferentes caracterísicas de modelos de restauração da Mata Atlântica. Dentre as características estudadas, a densidade básica da madeira foi determinada para diferentes espécies. $\mathrm{O}$ autor utilizou quatro amostras em diferentes alturas e encontrou a média de $0,49 \mathrm{~g} . \mathrm{cm}^{-3}$ para indivíduos de $S$. terebinthifolius em um plantio instalado em 1998, valor esse que se assemelha com os encontrados por BAGGIO (1998).

CAMPOE (2008) realizou uma pesquisa analisando o efeito de práticas silviculturais e outros fatores em plantios de restauração da Mata Atlântica. Os ENCICLOPÉDIA BIOSFERA, Centro Científico Conhecer - Goiânia, v.11 n.22; p. 836 2015 
ensaios foram realizados com indivíduos de 36 e 42 meses. Para a espécie $S$. terebinthifolius, o autor encontrou o valor médio de $0,44 \pm 0,02 \mathrm{~g} . \mathrm{cm}^{-3}$. Nota-se que este valor é mais próximo daquele encontrado neste estudo para o ápice, uma vez se trata de indivíduos mais jovens, cujos tecidos se aproximam mais da parte apical das plantas desta pesquisa.

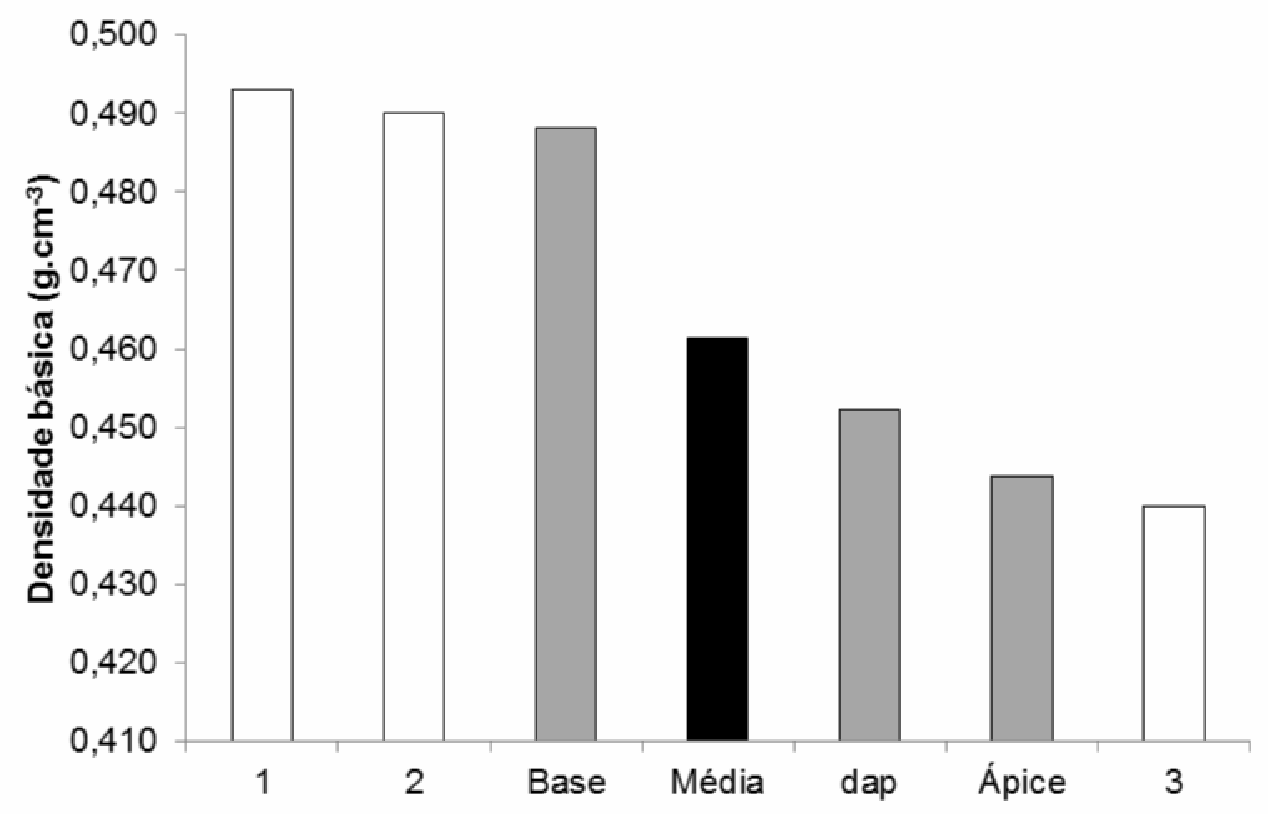

FIGURA 2 - Comparação da densidade básica da madeira de Schinus terebinthifolius neste estudo (em Pinhais-PR) e com outros estudos para a mesma espécie

Nota: Ref. 1: Baggio (1998); Ref. 2: Nogueira (2010); Ref. 3 : Campoe (2008).

Neste estudo não foi verificada uma relação significativa entre a densidade básica média e o diâmetro à altura do peito das árvores, ou seja, a densidade não varia com o porte das plantas (Figura 3 ). $O$ valor do coeficiente de correlação de Pearson foi de 0,407.

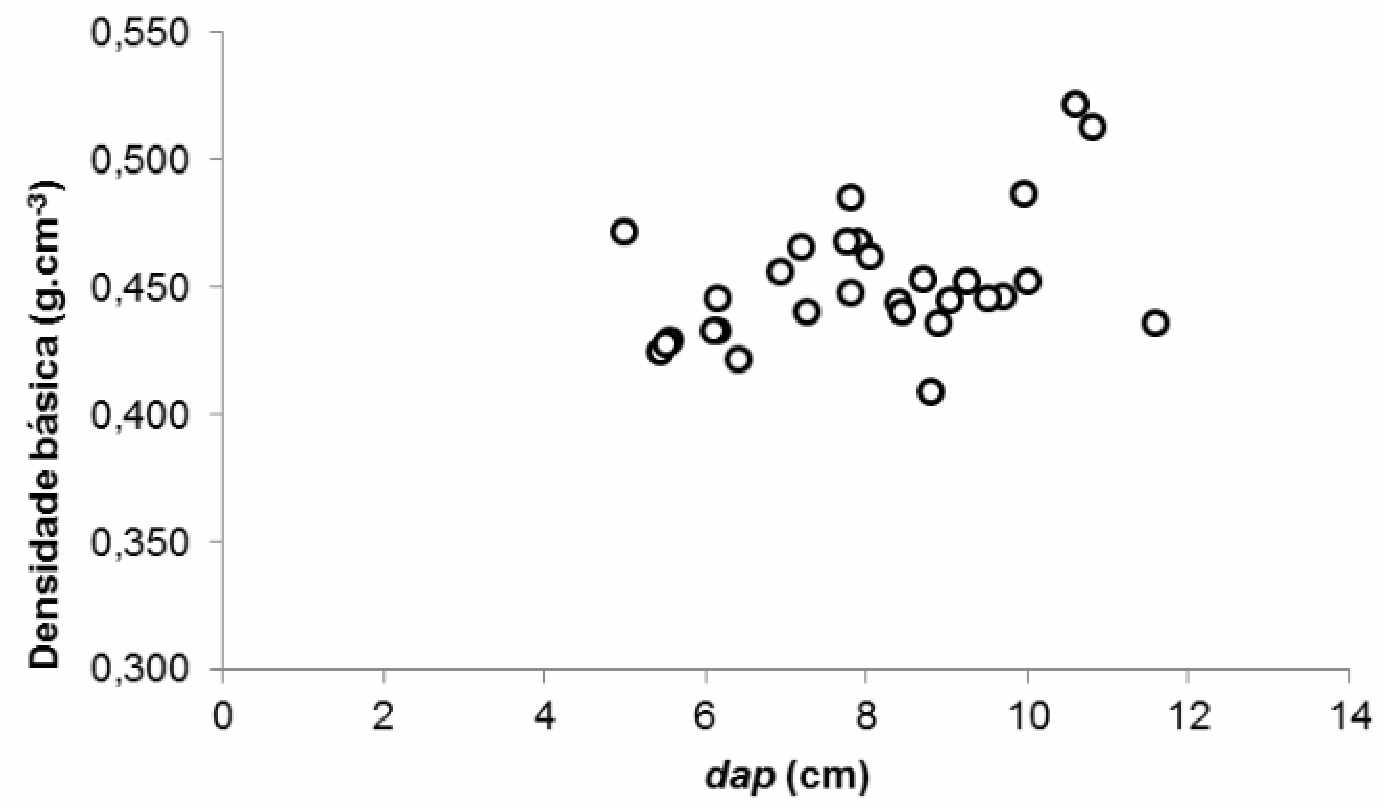


FIGURA 3 - Relação entre o diâmetro e densidade básica da madeira de 30 árvores de Schinus terebinthifolius em Pinhais-PR

Os valores mínimos e máximos de densidade na base foram de 0,415 e 0,575 g. $\mathrm{cm}^{-3}$, com maior concentração de valores entre $0,48 \mathrm{e} 0,50 \mathrm{~g} \cdot \mathrm{cm}^{-3}$. Já para a posição dap, os valores oscilaram entre 0,409 e $0,522 \mathrm{~g} \cdot \mathrm{cm}^{-3}$, com concentração na medida $0,46 \mathrm{~g} \cdot \mathrm{cm}^{-3}$. Para a posição ápice as densidades variaram de 0,396 a 0,530 $\mathrm{g} \cdot \mathrm{cm}^{-3}$, também com concentração na medida $0,46 \mathrm{~g} \cdot \mathrm{cm}^{-3}$.

A idade do indivíduo e as dimensões radiais e verticais do caule são variáveis importantes na construção de um valor médio para a densidade média de uma espécie (WILLIAMSON \& WIEMANN, 2010; SARMIENTO et al., 2011). Este estudo focou em indivíduos de uma única idade, plantados no ano de 2003 e em condições específicas de sítio e de manejo. As árvores em apreço foram plantadas no espaçamento de $2 \mathrm{~m} \times 2 \mathrm{~m}$ e o povoamento não foi desbastado, o que pode ser um fator que determine maior densidade, haja vista o limitado espaço vital. Com relação à altura, ou seja, a posição vertical, este estudo preocupou-se em determinar a densidade em três porções do fuste (base, dap e ponto de inversão morfológica, que correspondente ao ápice do tronco principal). Estudos em outras condições ambientais, que levem em conta também a variação radial da densidade são importantes para poder construir um valor médio da densidade mais abrangente para a espécie.

(a) Base

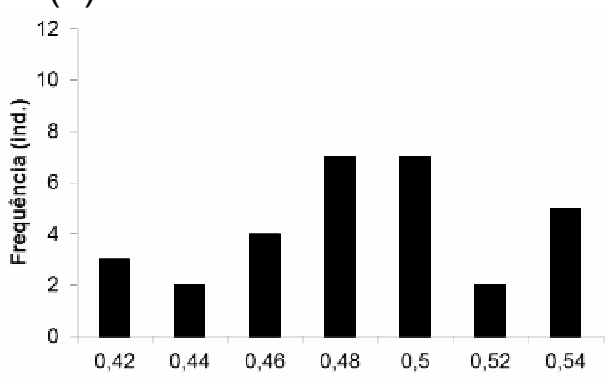

(b) Ápice

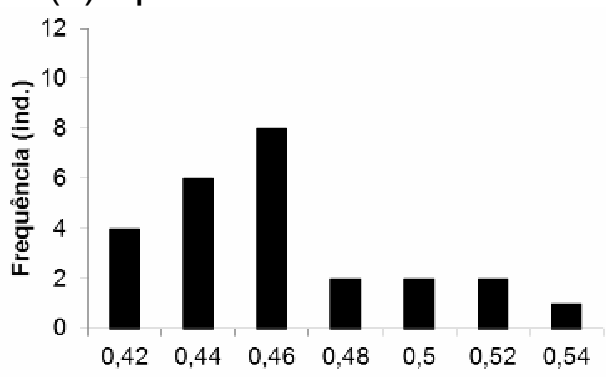

(b) dap

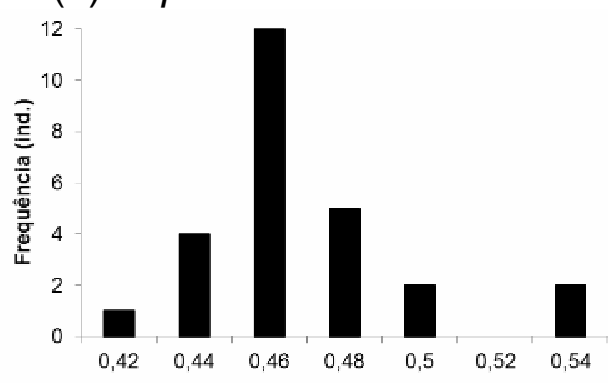


FIGURA 4 - Distribuição de frequência da densidade básica da madeira de 30 árvores de Schinus terebinthifolius em Pinhais-PR, em diferentes porções do fuste

\section{CONCLUSÕES}

- A aroeira é uma espécie florestal que possui densidade básica da madeira com valor intermediário: não é leve, nem pesada. Neste estudo o valor médio encontrado foi de $0,463 \mathrm{~g} \cdot \mathrm{cm}^{-3}$, comparável ao encontrado na literatura para plantas jovens dessa espécie;

- Houve diferença significativa entre a densidade básica da madeira na base em relação às medidas no dap e no ápice;

- Não há relação entre o dap e a densidade básica da madeira;

- A variabilidade da densidade básica é relativamente baixa, da ordem de $6 \%$ em termos de coeficiente de variação.

\section{REFERÊNCIAS}

ABNT - ASSOCIAÇÃO BRASILEIRA DE NORMAS TÉCNICAS. NBR 11941: madeira: determinação da densidade básica. Rio de Janeiro, 2003. 6p.

BAGGIO, A.J. Aroeira como potencial para usos múltiplos na propriedade rural. Boletim de Pesquisa Florestal, Colombo, n. 17, p. 25-32. 1988.

CAMPOE, O.C. Efeito de práticas silviculturais sobre a produtividade primária líquida de madeira, o índice de área foliar e a eficiência do uso da luz em plantios de restauração da Mata Atlântica. Dissertação (Mestrado em Ciências Recursos Florestais). Escola Superior de Agricultura Luiz de Queiroz. Universidade de São Paulo. 120p. 2008.

CARVALHO, P.E.R. Espécies arbóreas brasileiras. Brasília, DF: Embrapa Informação Tecnológica; Colombo: Embrapa Florestas, 2003, 1039p.

CARVALHO, M.G.; MELO, A.G.N.; ARAGÃO, C.F.S.; RAFFIN, F.N.; MOURA, T.F.A.L. Schinus terebinthifolius Raddi: chemical composition, biological properties and toxicity. Revista Brasileira de Plantas Medicinais, v.15, n.1, p.158-169. 2013.

CORADIN, L.; SIMINSKI, A.; REIS, A. Espécies nativas da flora brasileira de valor econômico atual ou potencial: plantas para o futuro - Região Sul, Brasília: MMA, 934p. 2011.

JATI, S.R.; FEARNSIDE, P.M.; BARBOSA, R.I. Densidade da madeira de árvores em savanas do norte da Amazônia brasileira. Acta Amazonica, v.44, n.1, p.79-86, 2014.

NOGUEIRA, L.R.J. Estoque de carbono na fitomassa e mudanças nos atributos do solo em diferentes modelos de restauração da Mata Atlântica. Tese (Doutorado em Ciências - Recursos Florestais). Escola Superior de Agricultura Luiz de Queiroz. Universidade de São Paulo. 94p. 2010. 
REITZ, R.; KLEIN, R.M.; REIS, A. Projeto madeira de Santa Catarina. Itajaí, Herbário Barbosa Rodrigues, 1978. 320p.

RIZZINI, C.T. Árvores e arbustos de cerrado. Rodriguésia, v. 26, n. 38, p. 63-77, 1970.

SANCHOTENE, M.C.C. Frutíferas nativas úteis à fauna na arborização urbana. Porto Alegre, Feplan, 1985. 311p.

SARMIENTO, C.; PATINO, S.; PAINE, C.E.; BEAUCHENE, J.; THIBAUT, A.; BARALOTO, C. Within-individual variation of trunk and branch xylem density in tropical trees. American Journal of Botany, v.98, p.140-149, 2011.

WILLIAMSON, G.B.; WIEMANN, M.C. Age-dependent radial increases in wood specific gravity of tropical pioneers in Costa Rica. Biotropica, v.42, p.590-597, 2010.

WILLIAMSON, G.B.; WIEMANN, M.C. Age versus size determination of radial variation in wood specific gravity: lessons from eccentrics. Trees, v.25, p.585-591. 2011. 